\title{
MODELING STOCHASTIC HYBRID SYSTEMS
}

\author{
Mrinal K. Ghosh * \\ Department of Mathematics, Indian Institute of Science, Bangalore - 560 012, India \\ mkg@math.iisc.ernet.in
}

Arunabha Bagchi

Department of Applied Mathematics, University of Twente, Post Box 217, 7500 AE Enschede, The Netherlands

a.bagchi@math.utwente.nl

\begin{abstract}
Stochastic hybrid systems arise in numerous applications of systems with multiple models; e.g., air traffc management, flexible manufacturing systems, fault tolerant control systems etc. In a typical hybrid system, the state space is hybrid in the sense that some components take values in a Euclidean space, while some other components are discrete. In this paper we propose two stochastic hybrid models, both of which permit diffusion and hybrid jump. Such models are essential for studying air traffic management in a stochastic framework.
\end{abstract}

Keywords: Stochastic hybrid systems, Markov processes, Ito-Skorohod type stochastic differential equations, hybrid jumps.

\section{Introduction}

In this article we study some classes of stochastic hybrid models. Stochastic hybrid systems arise in numerous applications of systems with multiple modes, e.g., flexible manufacturing systems, air traffic management, fault tolerant control systems etc. For various applications of stochastic hybrid systems we refer to [3], [8], [1], [11] and the references therein. In a typical hybrid system, the state space is hybrid in the sense that some components take values in a Euclidean space while some other

*Research supported by the IST project "HYBRIDGE", IST-2001-32460, of the European Commission 
components are discrete. The evolution of continuous and discrete components are intertwined in an intricate manner. This makes the analysis of a hybrid system quite involved and challenging. Several classes of stochastic hybrid systems have been studied in the literature, e.g., counting processes with diffusion intensity [10], [13], diffusion processes with Markovian switching parameters [11], [16], switching diffusions [8], [9], piecewise deterministic processes [5], [15], Markov decision drift processes [1]etc. All these stochastic hybrid systems arise in different kinds of applications.

Here we address two kinds of stochastic hybrid models. In the first model we construct a Markov process $(X(t), \theta(t))$, where $X(t) \in \mathbb{R}^{d}$ and $\theta(t) \in \Theta=\{1,2, \ldots, N\}$. Here $X(t)$ is governed by a stochastic differential equation of Ito-Skorohod type with drift coefficient, diffusion matrix and the 'jump' function depending on the discrete component $\theta(t)$. Thus $X(t)$ switches from one jump diffusion path to another as the discrete component $\theta(t)$ moves from one state to another. On the other hand, the discrete component $\theta(t)$ is a "controlled Markov chain" with a transition matrix that depends on the continuous component $X(t)$. A change in the discrete state $\theta(t)$ makes a switching in the continuous state. This apart the continuous state does jump at random times. At times this may lead to a situation where a switching triggers a jump and vice-versa. This model is discussed in the next section. Section 3 is devoted to the study of a very general stochastic hybrid system. The state of the system at time $t$, denoted by $(X(t), \theta(t))$ takes values in $\cup_{n}\left(S_{n} \times \Theta_{n}\right)$, where $\Theta_{n}=\left\{1,2, \ldots, N_{n}\right\}$ and $S_{n}$ is a subset of $\mathbb{R}^{d_{n}}$. Between the jumps $(X(t), \theta(t))$ is a switching diffusion. That is $\theta(\cdot)$ is a pure jump process taking values in $\Theta_{n}$; between successive jumps of $\theta(t), X(t)$ is a diffusion process. On the other hand, the infinitesimal jump rates of $\theta(t)$ depends on $X(t)$. Let $A_{n}$ be a subset of $S_{n}$. If $X(t)$ starting from some point in $S_{n}$, hits $A_{n}$ then it executes an instantaneous jump to some $S_{m}$. The destination of $X(t)$ at this moment is determined by a pre-determined map. The discrete component at this moment is also reset by a given map. We investigate a Markovian structure of this system by introducing another switching component in the systems.

A typical construction of a hybrid systems is based on stochastic differential equations driven by Wiener processes and Poisson random measures. For a comprehensive treatment of stochastic differential equation driven by Wiener processes and Poisson random measure we refer to [6], [7] and [12]. 


\section{Stochastic Hybrid Model I}

In this section we construct a Markov process $(X(t), \theta(t))$ taking values in $\mathbb{R}^{d} \times \Theta$ where $\Theta=\{1,2, \ldots, N\}$. The evolution of the process is governed by equations of the following form:

$$
\left.\begin{array}{c}
d X(t)=b(X(t), \theta(t)) d t+\sigma(X(t), \theta(t)) d W(t)+ \\
\int_{\mathbb{R}} g(X(t), \theta(t), u) p(d t, d u) \\
P(\theta(t+\delta t=j), \theta(t)=i, X(s), \theta(s), s \leq t) \\
=\lambda_{i j}(X(t)) \delta t+0(\delta t), i \neq j \\
X(0)=X_{0}, \theta(0)=\theta_{0} .
\end{array}\right\}
$$

Here $b, \sigma, g, \lambda$ are suitable functions, $\lambda_{i j} \geq 0, i \neq j, \sum_{j=1}^{N} \lambda_{i j}=0, W(\cdot)$ is a standard Wiener process and $p(\cdot, \cdot)$ is a certain Poisson random measure on $\mathbb{R}_{+} \times \mathbb{R}$ to be specified shortly. Under certain conditions we establish the existence of a pathwise unique solution of (1). We make certain assumptions on $b, \sigma, g, \lambda$. Let

$$
\begin{aligned}
b & : \mathbb{R}^{d} \times \Theta \rightarrow \mathbb{R}^{d} \\
\sigma & : \mathbb{R}^{d} \times \Theta \rightarrow \mathbb{R}^{d \times d} \\
g & : \mathbb{R}^{d} \times \Theta \times \mathbb{R} \rightarrow \mathbb{R} \\
\lambda_{i j} & : \mathbb{R}^{d} \rightarrow \mathbb{R}, i, j=1,2, \ldots, N .
\end{aligned}
$$

We make the following assumptions on the above functions.

(A1) For each $i=1,2, \ldots, N, b(\cdot, i)$ is bounded and Lipschitz continuous.

(A2) For each $i=1,2, \ldots, N, \sigma(\cdot, i)$ is bounded and Lipschitz continuous.

(A3) For $i, j=1,2, \ldots, N, \lambda_{i j}(\cdot)$ are bounded and measurable, $\lambda_{i j}(\cdot) \geq$ 0 for $i \neq j$, and $\sum_{j=1}^{N} \lambda_{i j}(\cdot)=0$.

(A4) Let $K_{1}$ be the support of $g(\cdot, \cdot, \cdot)$ and let $U_{1}$ be the projection of $K_{1}$ on $\mathbb{R}$. We assume that $U_{1}$ is bounded.

Note that in (1), the process $\theta(t)$ is a pure jump process. Thus by the results of $[6], \theta(t)$ may be represented by an integral with respect to a Poisson random measure. Following [4], [8], [9] we proceed to obtain this representation explicitly. To this end, we first embed $\Theta$ into $\mathbb{R}^{N}$ by identifying $i$ with $e_{i}$, the $i$ th unit vector in $\mathbb{R}^{N}$. For $i, j \in \Theta,: x \in \mathbb{R}^{d}$, 
let $\Delta_{i j}(x)$ be consecutive (with respect to the lexicographic ordering on $\Theta \times \Theta$ ) left closed, right open intervals on the real line, each having length $\lambda_{i j}(x)$. Define a function

$$
h: \mathbb{R}^{d} \times \Theta \times \mathbb{R} \rightarrow \mathbb{R}^{N}
$$

by

$$
h(x, i, u)=\left\{\begin{array}{ccc}
j-i & \text { if } & u \in \Delta_{i j}(x) \\
0 & \text { otherwise. }
\end{array}\right.
$$

Let $(X(t), \theta(t))$ be an $\mathbb{R}^{d} \times \Theta$-valued process given by the following stochastic differential equation of Ito-Skorohod type.

$$
\left.\begin{array}{c}
d X(t)=b(X(t), \theta(t)) d t+\sigma(X(t), \theta(t)) d W(t)+ \\
\int_{\mathbb{R}} g(X(t-), \theta(t-), u) p(d t, d u), \\
d \theta(t)=\int_{\mathbb{R}} h(X(t-), \theta(t-), u) p(d t, d u) \\
\text { for } t \geq 0, X(0)=X_{0}, \theta(0)=\theta_{0} .
\end{array}\right\}
$$

Here:

(i) $X_{0}$ is a prescribed $\mathbb{R}^{d}$-valued random variable.

(ii) $\theta_{0}$ is a given $\Theta$-valued random variable.

(iii) $W(\cdot)$ is a $d$-dimensional standard Wiener process.

(iv) $p(d t, d u)$ is a Poisson random measure with intensity $d t \times l(d u)$, where $l$ is the Lebesgue measure on $\mathbb{R}$.

By the construction of the function $h$ in (2), it is clear that a solution of (3) is also a solution of (1). Thus we prove the existence of an a.s. unique strong solution of (3). To achieve this we use the method in [6].

Theorem 1 Assume (A1)-(A4). Let $p(\cdot, \cdot), W(\cdot), X_{0}, \theta_{0}$ be independent. Then the equation (3) has an a.s. unique strong solution.

PROOF: Let $(\Omega, \mathcal{F}, P)$ be the underlying (complete) probability space on which $p(\cdot, \cdot), W(\cdot), X_{0}, \theta_{0}$ are defined. Let $\tilde{p}(\cdot)$ be the Poisson process on $(\Omega, \mathcal{F}, P)$ corresponding to the given Poisson random measure $p(\cdot, \cdot)$. Let $K_{2}=$ support of $h(\cdot, \cdot, \cdot)$ and $U_{2}$ the projection of $K_{2}$ on $\mathbb{R}$. By (A3), $U_{2}$ is a bounded set. Let $U=U_{1} \cup U_{2}$. Then $U$ is also bounded. Let $D_{\tilde{p}}$ denote the domain of the Poisson process $\tilde{p}(\cdot)$. Let

$$
D=\left\{t \in D_{\tilde{p}}: \tilde{p}(t) \in U\right\} .
$$


Since $l(U)<\infty, D$ is a discrete set in $(0, \infty)$ a.s. Let $\tau_{1}<\tau_{2}<\ldots<$ $\tau_{n}<\ldots$ be the enumeration of all elements in $D$. Let

$$
\mathcal{F}_{t}=\sigma\{W(s), p(A, B) \mid s \leq t, A \in \mathcal{B}([0, t]), B \in \mathcal{B}(\mathbb{R})\}
$$

Then it is easy to see that $\tau_{n}$ is an $\mathcal{F}_{t^{-}}$stopping time for each $n$ and $\tau_{n} \uparrow \infty$ a.s. First we establish the existence and uniqueness of the solution in the time interval $\left[0, \tau_{1}\right]$. To achieve this consider the following stochastic differential equation:

$$
Y(t)=X_{0}+\int_{0}^{t} b\left(Y(s), \theta_{0}\right) d t+\sigma\left(Y(s), \theta_{0}\right) d W(s) .
$$

First assume $X_{Q}=x \in \mathbb{R}^{d}$ and $\theta_{0}=i \in \Theta$ for some $x, i$. Under (A1), (A2), the equation (4) has an a.s. unique strong solution which depends measurably on $x i,:$ and : $W(\cdot)$. The solution for the initial condition $X_{0}, \theta_{0}$ is obtained by replacing $(x, i)$ by $\left(X_{0}, \theta_{0}\right)$. Now set

$$
\begin{gathered}
X_{1}(t)=\left\{\begin{array}{l}
Y(t) \text { if } 0 \leq t<\tau_{1} \\
Y\left(\tau_{1}-\right)+g\left(Y\left(\tau_{1}-\right), \theta_{0}, \tilde{p}\left(\tau_{1}\right)\right) \text { if } t=\tau_{1}
\end{array}\right. \\
\theta_{1}(t)=\left\{\begin{array}{l}
\theta_{0} \text { if } 0 \leq t<\tau_{1} \\
\theta_{0}+h\left(Y\left(\tau_{1}-\right), \theta_{0}, \tilde{p}\left(\tau_{1}\right)\right) \text { if } t=\tau_{1} .
\end{array}\right.
\end{gathered}
$$

The process $\left\{X_{1}(t), \theta_{1}(t)\right\}_{t \in\left[0, \tau_{1}\right]}$ is clearly the unique solution of (3) in the time interval $\left[0, \tau_{1}\right]$. Next, let $\tilde{X}=X_{1}\left(\tau_{1}\right), \tilde{\theta}=\theta\left(\tau_{1}\right),: \tilde{W}(\cdot)=$ $\tilde{W}\left(\cdot+\tau_{1}\right)-W\left(\tau_{1}\right)$, and $\hat{p}=(\hat{p}(t))$, where $D_{\hat{p}}=\left\{s: s+\tau_{1} \in D_{\tilde{p}}\right\}$ and $\hat{p}(s)=\tilde{p}\left(s+\tau_{1}\right)$. Proceeding as before we can determine the process $\left(\tilde{X}_{2}(t), \tilde{\theta}_{2}(t)\right)$ on $\left[0, \hat{\tau}_{1}\right]$ with respect to $\tilde{X}, \tilde{\theta}, \tilde{W}$ and $\hat{p}$. Clearly $\hat{\tau}_{1}=$ $\tau_{2}-\tau_{1}$. Define $(X(t), \theta(t))$ by

$$
(X(t), \theta(t))=\left\{\begin{array}{l}
\left(X_{1}(t), \theta_{1}(t)\right) \quad \text { if } t \in\left[0, \tau_{1}\right] \\
\left(\tilde{X}_{2}\left(t-\tau_{1}\right), \tilde{\theta}_{2}\left(t-\tau_{1}\right)\right) \text { if } t \in\left[\tau_{1}, \tau_{2}\right] .
\end{array}\right.
$$

It is now clear that $(X(t), \theta(t))$ is the unique solution of (3) in the interval $\left[0, \tau_{2}\right]$. Proceedings this way $(X(t), \theta(t))$ is determined uniquely in $\left[0, \tau_{n}\right]$ for every $n$. Hence a.s. $(X(t), \theta(t))$ is determined uniquely for all time. 


\section{Some comments are in order.}

\section{Remark 2.1}

(i) The boundedness assumption on $b$ and $\sigma$ in (A1), (A2) may be relaxed. It may be replaced by a growth condition of the following type: there exists a constant $C$ such

$$
\|b(x, i)\|^{2}+\|\sigma(x, i)\|^{2} \leq C\left(1+\|x\|^{2}\right) .
$$

Similarly the Lipschitz continuity assumption in (A1), (A2) may be replaced by locally Lipschitz continuity.

(ii) If for each $i=1,2, \ldots, N, \sigma(\cdot, i) \sigma^{*}(\cdot, i)$ is uniformly elliptic, i.e., the least eigenvalue of $\sigma(\cdot, i) \sigma^{*}(\cdot, i)$ is uniformly bounded away from zero, then we can drop any kind of continuity assumption on $b(\cdot, i)$. In fact if $b(\cdot, i)$ is bounded and measurable and $\sigma(\cdot, i)$ is bounded and Lipschitz and (A3), (A4) hold, then under the uniform ellipticity condition it can be shown as in [8], [9], (3) has an a.s. unique strong solution.

(iii) It is clear from the construction that the process $(X(t), \theta(t))$ is Markov. Let $\mathcal{L}$ denote the extended generator of $(X(t), \theta(t))$. Then for $f \in C^{2}\left(\mathbb{R}^{d} \times \Theta\right) \subset D(\mathcal{L})$, it can be shown that

$$
\mathcal{L}: f(x, i)=L_{i} f(x, i)+\sum_{j=1}^{N} \int_{\mathbb{R}^{d}}[f(y, j)-f(x, i)] \nu_{x, i}(d y \times\{j\})
$$

where

$$
L_{i} f(x, i)=\sum_{k=1}^{d} b_{k}(x, i) \frac{\partial f(x, i)}{\partial x_{k}}+\frac{1}{2} \sum_{j, k=1}^{d} \sum_{l=1}^{d} \sigma_{j l}(x, i) \sigma_{k l}(x, i) \frac{\partial^{2} f(x, i)}{\partial x_{j} \partial x_{k}}
$$

and

$\nu_{x, i}(A \times\{j\})=\int_{\mathbb{R}} I_{A \times\{j\}}(x+g(x, i, u), i+h(x, i, u)) d u$ for $A \in \mathcal{B}\left(\mathbb{R}^{d}\right)$.

(iv) Note that the times at which jumps or switchings occur are determined by the stopping times $\tau_{n}, n=1,2, \ldots$ But at every $\tau_{n}$, a jump or a switching may not occur. For example if at $t=\tau_{1}, g\left(Y\left(\tau_{1}-\right), \theta_{0}, \tilde{p}\left(\tau_{1}\right)\right)=$ 0 , there is no jump in the trajectory of $X(t)$ at this time. Similarly, if $h\left(Y\left(\tau_{1}-\right), \theta_{0}, \tilde{p}\left(\tau_{1}\right)\right)=0$, then $\theta(t)$ remains at $\theta_{0}$ and thus there is no switching in the trajectory of $X(t)$ at this time. If $g\left(Y\left(\tau_{1}-\right), \theta_{0}, \tilde{p}\left(\tau_{1}\right)\right) \neq$ 0 but $h\left(Y\left(\tau_{1}-\right), \theta_{0}, \tilde{p}\left(\tau_{1}\right)=0\right.$, then there will be a jump at $t=\tau_{1}$, but no switching at $t=\tau_{1}$. Similarly if $g\left(Y\left(\tau_{1}-\right), \theta_{0}, \tilde{p}\left(\tau_{1}\right)\right)=0$, but $h\left(Y\left(\tau_{1}-\right), \theta_{0}, \tilde{p}\left(\tau_{1}\right)\right) \neq 0$, there is no jump but only a switching occurs at $t=\tau_{1}$. On the other hand if $g\left(Y\left(\tau_{1}-\right), \theta_{0}, \tilde{p}\left(\tau_{1}\right) \neq 0\right.$ and 
$h\left(Y\left(\tau_{1}-\right), \theta_{0}, \tilde{p}\left(\tau_{1}\right)\right) \neq 0$ there is a simultaneous jump and switching at $t=\tau_{1}$. This kind of mechanism goes on for $t>\tau_{1}$.

(v) We now focus our attention to a specific case where jumps and switching always occur simultaneously. Let

$$
\tilde{g}: \mathbb{R}^{d} \times \Theta \rightarrow \mathbb{R}^{d}
$$

be a function which is bounded and measurable. Let the function $g(\cdot, \cdot, \cdot)$ be given by

$$
g(x, i, u)=\left\{\begin{array}{lll}
\tilde{g}(x, j) & \text { if } & u \in \Delta_{i j}(x) \\
0 & \text { otherwise. }
\end{array}\right.
$$

If $g(\cdot, \cdot, \cdot)$ is of the above form, then from (2) and (7), it is clear that the jumps and switchings always occur together. In this specific case the extended generator of $(X(t), \theta(t))$ can be expressed explicitly in terms of $b, \sigma, \tilde{g}$ and $\lambda_{i j}$. Let $\mathcal{L}$ denote the extended generator of $(X(t), \theta(t))$. Let $f: \mathbb{R}^{d} \times \Theta \rightarrow \mathbb{R}$ be a smooth function. Then using Ito's formula one can show that

$$
\mathcal{L}: f(x, i)=L_{i} f(x, i)+\sum_{j=1}^{N} \lambda_{i, j}(x)(f(x+g(x, i))-f(x, i)
$$

where $L_{i} f(x, i)$ is as in (6).

(vi) Consider the non-degenerate case, i.e., when for each $i$

$$
\sigma(\cdot, i) \sigma^{*}(\cdot, i)
$$

is uniformly elliptic. In this case if for each $i, b(\cdot, i)$ is bounded and measurable, and $\sigma(\cdot, i)$ is bounded and Lipschitz continuous, (A3) holds and $g(\cdot, \cdot, \cdot)$ is of the form (7), then one can show as in [9] that the process $(X(t), \theta(t))$ is strong Feller.

\section{Stochastic Hybrid Model II}

In this section we study a very general stochastic hybrid system. We refer to [2], [14]for analogous controlled stochastic hybrid systems. The state of the system at time $t$, denoted by $(X(t), \theta(t))$, takes values in $\cup_{n=1}^{\infty}\left(S_{n} \times \Theta_{n}\right)$, where $\Theta_{n}=\left\{1,2, \ldots, M_{n}\right\}$ and $S_{n}$ is a subset of $\mathbb{R}^{d_{n}}$. 
Between the jumps of $X(t)$ the state equations are of the form

$$
\begin{aligned}
& d X(t)=b^{n}(X(t), \theta(t)) d t+\sigma^{n}(X(t), \theta(t)) d W^{n}(t) \\
& P(\theta(t+\delta t)=j \mid \theta(t)=i, X(s), \theta(s), s \leq t) \\
& =\lambda_{i j}^{n}(X(t)) \delta t+0(\delta t), i \neq j, \\
& X(0)=X_{0}, \theta(0)=\theta_{0},
\end{aligned}
$$

where for each $n \in \mathbb{N}$

$$
\begin{aligned}
& b^{n}: S_{n} \times \Theta_{n} \rightarrow \mathbb{R}^{d_{n}} \\
& \sigma^{n}: S_{n} \times \Theta_{n} \rightarrow \mathbb{R}^{d_{n} \times d_{n}} \\
& \lambda_{i j}^{n}: S_{n} \rightarrow \mathbb{R}
\end{aligned}
$$

are suitable functions, $\lambda_{i j}^{n}(\cdot) \geq 0, i \neq j,: \sum_{j=1}^{M_{n}} \lambda_{i j}^{n}(\cdot)=0,: X_{0}, \theta_{0}$ are $S_{n}-$ and $\Theta_{n}-$ valued random variables, and $W^{n}(\cdot)$ is a standard $d_{n^{-}}$ dimensional Wiener process. For each $n \in \mathbb{N}$, let $A_{n} \subset S_{n},: D_{n} \subset$ $S_{n}$. The set $A_{n}$ is the set of instantaneous jump, whereas $D_{n}$ is the destination set. If at some random time $X(t)$ hits $A_{n}$, then it executes an instantaneous jump. The destination of $(X(t), \theta(t))$ at this juncture is determined by a map

$$
g_{n}: A_{n} \times \Theta_{n} \rightarrow \cup_{m}\left(D_{m} \times \Theta_{m}\right) .
$$

After reaching the destination, the process $(X(t), \theta(t))$ follows the same evolutionary mechanism over and over again.

To ensure the existence of such a pair of processes we need to make certain assumptions.

For each $n \in \mathbb{N}$, let $S_{n}$ be the closure of a connected open subset of some Euclidean space $\mathbb{R}^{d_{n}}$. For each $n \in \mathbb{N}, A_{n}$ and $D_{n}$ are closed, and $A_{n} \cap D_{n}=\phi$.

We now make the following assumptions.

(A5) For each $n \in \mathbb{N}$ and $i \in \Theta_{n}, b^{n}(\cdot, i)$ is Lipschitz continuous.

(A6) For each $n \in \mathbb{N}$ and $i \in \Theta_{n}, \sigma^{n}(\cdot, i)$ is Lipschitz continuous.

(A7) For each $n \in \mathbb{N}, i, j \in \Theta_{n}, \lambda_{i j}^{n}(\cdot)$ are bounded and measurable.

(A8) The maps $g_{n}, n \in \mathbb{N}$, are bounded and uniformly continuous.

(A9) $\inf _{n} d\left(A_{n}, D_{n}\right)>0$. 
Let $(\Omega, \mathcal{F}, P)$ be the underlying (complete) probability space on which $W^{n}(\cdot), X_{0}, \theta_{0}$ are defined. As in the previous section $\theta(t)$ can be expressed as an integral with respect to a Poisson random measure. Let $p(\cdot, \cdot)$ be $\mathbb{R}_{+} \times \mathbb{R}$-valued Poisson random measure with the intensity $d t \times l(d u)$ as in the previous section. Construct the maps

$$
h^{n}: \mathbb{R}^{d_{n}} \times \Theta_{n} \times \mathbb{R} \rightarrow \mathbb{R}^{M_{n}}
$$

as in the previous section such that

$$
d \theta(t)=\int_{\mathbb{R}} h^{n}(X(t-), \theta(t-), u) p(d t, d u) .
$$

Let

$$
\mathcal{F}_{t}^{n}=\sigma\left\{W^{n}(s), p(A, B) \mid s \leq t, A \in \mathcal{B}([0, t]), B \in \mathcal{B}(\mathbb{R})\right\} .
$$

Let $X_{0}, \theta_{0}, W^{n}(\cdot), p(\cdot, \cdot)$ be independent. Then as in the previous section, we can show that under (A5), (A6) and (A7), the equation (8) has an a.s. unique strong solution, denoted by $\left(X^{n}(t), \theta^{n}(t)\right)$ which takes values in $\mathbb{R}^{d_{n}} \times \Theta_{n}$. Let

$$
\tau_{1}=\inf \left\{t \geq 0 \mid: X(t) \in A_{n}\right\} .
$$

Then $\tau_{1}$ is an $\mathcal{F}_{t}^{n}$ stopping time. Now define the process $(X(t), \theta(t))$ by

$$
(X(t), \theta(t))=\left\{\begin{array}{l}
\left(X^{n}(t), \theta^{n}(t)\right), \quad 0 \leq t<\tau_{1} \\
g_{n}\left(X^{n}\left(\tau_{1}-\right), \theta^{n}\left(\tau_{1}-\right)\right), \quad t=\tau_{1} .
\end{array}\right.
$$

Note that $\left(X\left(\tau_{1}\right), \theta\left(\tau_{1}\right)\right) \in D_{m} \times \Theta_{m}$, for some $m \in \mathbb{N}$. From $\tau_{1}$ on the system continues with the same mechanism from the state $\left(X\left(\tau_{1}\right), \theta\left(\tau_{1}\right)\right)$. Let

$$
\mathcal{F}_{t}=\vee_{n} \mathcal{F}_{t}^{n}
$$

Thus there is a sequence of $\mathcal{F}_{t}$ stopping times $0=\tau_{0} \leq \tau_{1}<\tau_{2}<\tau_{3}<$ $\ldots<\tau_{m}<\ldots$ such that $\tau_{m} \uparrow \infty$ a.s. and in the interval $\left[\tau_{m}, \tau_{m+1}\right)$, : $m=0,1, \ldots$ the process $(X(t), \theta(t))$ evolves according to (8) for some index $n \in \mathbb{N}$. At times $\tau_{m}, m \geq 1$, there is an instantaneous jump determined by the map $g_{m}$.

Note that, though in each interval of the type $\left[\tau_{m}, \tau_{m+1}\right)$, the evolution of $(X(t), \theta(t))$ follows a Markovian type dynamics, the process $(X(t), \theta(t)),: t \in[0, \infty)$, is not a Markov process. This is because we have not thus far accounted for a dynamical variable $\eta(t)$, to be introduced shortly, which is intricately linked with the evolution of $(X(t), \theta(t))$. Let $\eta(t)$ be an $\mathbb{N}$ valued process defined by

$$
\eta(t)=n \text { if }(X(t), \theta(t)) \in S_{n} \times \Theta_{n} .
$$


The process $\eta(t)$ is a piecewise constant process, it changes from $n$ to $m$ when $(X(t), \theta(t))$ jumps from the regime $S_{n} \times \Theta_{n}$ to the regime $S_{m} \times \Theta_{m}$. Thus $\eta(t)$ is an indicator of a regime and a change in $\eta(t)$ means a switching in the regimes in which $(X(t), \theta(t))$ evolves. One can show that the process $(X(t), \theta(t), \eta(t))$ is Markov. To see this more clearly we investigate the equations governing the process $(X(t), \theta(t), \eta(t))$. To this end, let

$$
\begin{aligned}
& \tilde{S}=\left\{(x, i, n) \mid x \in S_{n}, \quad i \in \Theta_{n}\right\} \\
& \tilde{A}=\left\{(x, i, n) \mid x \in A_{n}, \quad i \in \Theta_{n}\right\} \\
& \tilde{D}=\left\{(x, i, n) \mid x \in D_{n}, \quad i \in \Theta_{n}\right\}
\end{aligned}
$$

Clearly $(X(t), \theta(t), \eta(t))$ is an $\tilde{S}$-valued process. The set $\tilde{A}$ is the set where jumps occur and $D$ is the destination set for this process. The sets $\cup_{n}\left(S_{n} \times \Theta_{n}\right), \cup_{n}\left(A_{n} \times \Theta_{n}\right)$ and $\cup_{n}\left(D_{n} \times \Theta_{n}\right)$ can be embedded in $\tilde{S}, \tilde{A}$ and $\tilde{D}$ respectively.

Let $d^{0}$ denote the injection map of $\bigcup_{n}\left(D_{n} \times \Theta_{n}\right)$ into $\tilde{D}$. Define three maps $\tilde{g}_{i}: \tilde{A} \rightarrow \tilde{D},: i=1,2,:: \tilde{h}: \tilde{A} \rightarrow \mathbb{N}$

$$
\left.\begin{array}{l}
\tilde{g}_{1}(x, i, n)=\text { the first component in } d^{0}\left(g_{n}(x, i)\right) \\
\tilde{g}_{2}(x, i, n)=\text { the second component in } d^{0}\left(g_{n}(x, i)\right) \\
\tilde{h}(x, i, n)=\text { the third argument in } d^{0}\left(g_{n}(x, i)\right) .
\end{array}\right\}
$$

To describe the evolution of $(X(t), \theta(t), \eta(t))$ there is a sequence of $\mathcal{F}_{t}$ stopping times

$$
\tau_{1}<\tau_{2}<\tau_{3}<\ldots<\tau_{m}<\ldots
$$

$\tau_{m} \uparrow \infty$ a.s. which are the successive hitting times of $\tilde{A}$, such that for $t=\tau_{m}$

$$
\left.\begin{array}{rl}
\left(X\left(\tau_{m}\right), \theta\left(\tau_{m}\right)\right)= & \left(\tilde{g}_{1}\left(X\left(\tau_{m}-\right), \theta\left(\tau_{m}-\right), \eta\left(\tau_{m}-\right)\right),\right. \\
& \left.\tilde{g}_{2}\left(X\left(\tau_{m}-\right), \theta\left(\tau_{m}-\right), \eta\left(\tau_{m}-\right)\right)\right) \\
\eta\left(\tau_{m}\right)=\tilde{h}\left(X\left(\tau_{m}-\right), \theta\left(\tau_{m}-\right), \eta\left(\tau_{m}-\right)\right),
\end{array}\right\}
$$

where $\tilde{g}_{i}, \tilde{h}$ are defined in (13). For $\tau_{m}<t<\tau_{m+1}$

$$
\left.\begin{array}{l}
d X(t)=b(X(t), \theta(t), \eta(t)) d t+\sigma(X(t), \theta(t)), \eta(t)) d W^{\eta(t)}(t) \\
d \theta(t)=\int_{\mathbb{R}} h(X(t-), \theta(t-), \eta(t-) u) p(d t, d u)
\end{array}\right\}
$$

where $b(x, i, n)=b^{n}(x, i), \sigma(x, i, n)=\sigma^{n}(x, i), h(x, i, n, u)=h^{n}(x, i, u)$. 
The stopping time $\tau_{m+1}$ is defined by

$$
\tau_{m+1}=\inf \left\{t>\tau_{m} \mid(X(t-), \theta(t-), \eta(t-)) \in \tilde{A}\right\} .
$$

The equations for $(X(t), \theta(t), \eta(t))$ may thus be summarized as follows:

$$
\begin{aligned}
d X(t) & =[b(X(t), \theta(t), \eta(t)) \\
& \left.+\sum_{m=0}^{\infty}\left[\tilde{g}_{1}\left(X\left(\tau_{m}-\right), \theta\left(\tau_{m}-\right), \eta\left(\tau_{m}-\right)\right)-X\left(\tau_{m}-\right)\right] \delta\left(t-\tau_{m}\right)\right] d t \\
& +\sigma(X(t), \theta(t), \eta(t)) d W^{\eta(t)}(t), \\
d \theta(t) & =\int_{\mathbb{R}} h(X(t-), \theta(t-), \eta(t-) u) p(d t, d u) \\
& \left.+\sum_{m=0}^{\infty}\left[\tilde{g}_{2}\left(X\left(\tau_{m}-\right), \theta\left(\tau_{m}-\right), \eta\left(\tau_{m}-\right)\right)-\theta\left(\tau_{m}-\right)\right] \delta\left(t-\tau_{m}\right)\right] d t \\
d \eta(t) & =\sum_{m=0}^{\infty}\left[\tilde{h}\left(X\left(\tau_{m}-\right), \theta\left(\tau_{m}-\right), \eta\left(\tau_{m}-\right)\right)-\eta\left(\tau_{m}-\right)\right] I_{\left\{\tau_{m} \leq t\right\}}
\end{aligned}
$$

where $\delta$ is the Dirac measure.

From the above equation it is clear that the $\tilde{S}$-valued process

$$
(X(t), \theta(t), \eta(t))
$$

is a Markov process. Note that the stochastic hybrid model constructed in this section generalizes the stochastic hybrid models studied in [2], [14]. In the stochastic hybrid model studied in [2], there is no discrete component like $\theta(t)$. In [14] the discrete component $\theta(t)$ is included, but this component remains unchanged when the continuous component $X(t)$ makes an instantaneous jump. In our model we have removed this restriction on the dynamics of $\theta(t)$, and allow it to change when $X(t)$ changes. Thus we automatically have simultaneous jumps and switchings. Moreover in [14], the same functions $b, \sigma, \lambda_{i j}$ are used in every component of the state space $S_{n} \times \Theta_{n}$, whereas in our model, these functions depend on the index $n$. Thus our dynamics are more general than the one treated in [14]. Hence we have constructed a stochastic hybrid model which is more general than the models in [2] and [14].

\section{Conclusion}

In this paper we have explicitly constructed two stochastic hybrid systems. We established the existance and uniqueness of a strong solution 
in both cases, and showed that both solutions are Markov processes. The important point is that both models allow for simultaneous jumps in the trajectory and model parameters, the "so-called" case of a hybrid jump.

\section{Acknowlegments}

We are grateful to Dr. H. Blom of NLR (Amsterdam) for stimulating discussions during the course of this research.

\section{References}

[1] F. A. van der Duyn Schouten and A. Hordijk. Average optimal policies in markov decision drift processes with applications to a queueing and a replacement model. Adv. Appl. Prob., 15:274-303, 1983.

[2] A. Bensoussan and J. L. Menadi. Stochastic hybrid control. J.Math. Anal. Appl., 249:261-268, 2000.

[3] H. A. P. Blom. Bayesian estimation for decision-directed stochastic control. $\mathrm{PhD}$ thesis, Delft Univ. of Technology, 1990.

[4] R. W. Brockett and G. L. Blankenship. A representation theorem for linear differential equations with markovian switching. Proc. Allerton Conf. Circ. Syst. Th., pages 671-679, 1977.

[5] M. H. A. Davis. Markov Moldels and Optimisation. Chapman and Hall, 1999.

[6] N. Ikeda and S. Watanabe. Stochastic Differential Equations and Diffusion Processes, Second Edition. North-Holland, Kodansha, 1989.

[7] J. Jacod and A. N. Shiryayev. Limit Theorems for Stochastic Processes. Springer-Verlag, 1980.

[8] A. Arapostathis, M. K. Ghosh and S. I. Marcus. Optimal control of switching diffusions with application to flexible manufacturing systems. SIAM J. Control Optim., 31:1183-1204, 1993.

[9] A. Arapostathis, M. K. Ghosh and S. I. Marcus. Ergodic control of switching diffusions. SIAM J. Control Optim., 35:1952-1988, 1997.

[10] S. I. Marcus. Average optimal policies in markov decision drift processes with applications to a queueing and a replacement model. Adv. Appl. Prob., 15:274303, 1983.

[11] M. Mariton. Jump Linear Systems in Automatic Control. Marcel Dekker, 1990.

[12] A. V. Skorohod. Asymptotic Methods in the Theory of Stochastic Differential Equations. AMS, 1989.

[13] D. L. Snyder. Random Point Processes. Wiley, 1975.

[14] M. K. Ghosh, V. S. Borkar and P. Sahay. Optimal control of a stochastic hybrid system with discounted cost. J. Optim. Theory Appl., 101:557-580, 1991.

[15] D. Vermes. Optimal control of piecewise deterministic processes. Stochastics, 14:165-207, 1985.

[16] W. M. Wonham. Random differential equations in control theory. Probability Analysis in Applied Mathematics, vol. 2, A. T. Bharucha-Reid (Editor). Academic Press, 1970. 